\title{
Effect of dietary fatty acid saturation on egg production at end-of-lay
}

\author{
G.C. Buitendach ${ }^{1}$, F.H. de Witt ${ }^{1 \#}$, A. Hugo ${ }^{2}$, H.J. van der Merwe ${ }^{1}$ \& M.D. Fair ${ }^{1}$ \\ ${ }^{1}$ Department of Animal, Wildlife and Grassland Science \\ ${ }^{2}$ Department of Microbial, Biochemical and Food Biotechnology \\ P.O. Box 339, University of the Free State, Bloemfontein 9300, South Africa
}

(Received 8 December 2012; Accepted 26 September 2013; First published online 11 October 2013)

\begin{abstract}
Copyright resides with the authors in terms of the Creative Commons Attribution 2.5 South African Licence. See:
http://creativecommons.org/licenses/by/2.5/za/Condition of use: The user may copy, distribute, transmit and adapt the work, but must recognise the authors and the South African Journal of Animal Science
\end{abstract}

\begin{abstract}
This study was conducted to investigate the effects of dietary fatty acid saturation on production performances of laying hens at end-of-lay. Five isoenergetic (12.6 MJ AME/kg DM) and isonitrogenous (170 g CP/kg DM) diets were formulated using different lipid sources at a constant $30 \mathrm{~g} / \mathrm{kg}$ inclusion level. The control diet was formulated using a blend (50:50) of linseed- and fish oil, while the other treatments consist of pure fish oil (polyunsaturated n-3), sunflower oil (polyunsaturated n-6), high oleic acid (HO) sunflower oil (monounsaturated n-9) and tallow (SFA). Two hundred, individually caged Hy-Line SilverBrown laying hens (20 weeks of age) were randomly allocated to the five dietary treatments ( $\mathrm{n}=40$ replicates/treatment) and received the experimental diets for 54 weeks from 20 to 74 weeks of age. During weeks 58, 62, 66, 70 and 74 of age (end-of-lay period), all eggs produced were recorded and individually weighed while feed intake, as well as body weights of birds, were determined. Data for the respective collection weeks were pooled to calculate and statistical analyse production parameter means for the end-oflay period. Average daily feed intake of birds in the polyunsaturated n-3 treatment $(97.5 \mathrm{~g} / \mathrm{b} / \mathrm{d})$ were the lowest while that of the polyunsaturated n-6 treatment $(102.4 \mathrm{~g} / \mathrm{b} / \mathrm{d})$ the highest. Furthermore, despite the significant effect of fatty acid saturation on feed intake, it had no effect on hen-day egg production, egg weight, egg output, feed efficiency or body weight of hens during end-of-lay. Since results of the current study fail to indicate a clear trend regarding dietary fatty acid saturation on feed intake of birds, it could be concluded that the long term exposure to a range of fatty acid saturation levels, has no negative effect on hen performance.
\end{abstract}

Keywords: Eicosapentoenoic-, docosahexaenoic-, $\alpha$-linolenic-, linoleic acid

"Corresponding author: dewittfh@ufs.ac.za

\section{Introduction}

Eggs are a valuable source of protein and contain many substances with biological functions beyond nutrition (Ceylan et al., 2011). However, the major drawback of eggs as a food source for human consumption is its cholesterol content. The correlations between dietary cholesterol content, blood low density lipoprotein (LDL) cholesterol and atherosclerosis have been recognised for some years (McNamara, 2000; Simopoulos, 2000). Nevertheless, controlled metabolic studies conducted with humans revealed that the effect of dietary cholesterol on blood LDL cholesterol is relatively insignificant compared to that of saturated and trans-fatty acids (Hu et al., 1999). Alternatively, unsaturated fatty acids (UFAs) are known to reduce high blood LDL cholesterol levels that are caused by the above mentioned factors (McNamara, 2000). This "beneficial” function of UFAs should be of critical importance in the choice of food sources for people affected by conditions such as high blood LDL levels and -cholesterol. The relationship between cholesterol and cardio vascular disease (CVD) is one of the main reasons why health practitioners advise patients to try and avoid food with high levels of cholesterol and/or saturated fats. However, Leskanich \& Noble (1997), McNamara (2000) and Simopoulos (2000) illustrated that CVD is related to the fatty acid composition of the specific food source, rather than to the cholesterol content itself. Consumption of the correct ratio of omega- 
6 to omega-3 (n-6 : n-3) fatty acids, as well as the presence of sufficient essential fatty acids (EFAs), could reduce the risk of CVD in humans (Surai et al., 2000; McNamara, 2000; Simopoulos, 2000).

It is known that eggs are naturally low in $\alpha$-linolenic acid and do not contain eicosapentoenoic (EPA) and docosahexaenoic (DHA) fatty acids (Souza et al., 2008). Fortunately, it is possible to overcome these deficiencies by means of dietary enrichment with essential fatty acids. Several reports (Scheideler \& Froning, 1996; Surai et al., 2000; González-Muñoz et al., 2009, Ceylan et al., 2011) revealed the hen’s unique ability to directly deposit dietary lipids into the egg yolk, creating an opportunity to modify the yolk fatty acid profile into a possible source of UFAs, depending on the dietary treatment. Scheideler \& Froning (1996) indicated that the use of supplemental dietary fat in commercial poultry diets has been demonstrated to improve growth, feed efficiency, egg size, egg yield and hatchability in poultry. In addition to their recognised value as a dense energy source, supplemental fats are excellent sources of essential fatty acids (EFAs), enhance the absorption of fat-soluble vitamins, increase the palatability of the diet and reduce dustiness of the feed (Celebi \& Macit, 2008). Many studies (Scheideler \& Froning, 1996; Cachaldora et al., 2006; Cachaldora et al., 2008; Souza et al., 2008) investigated dietary fatty acid saturation in the production of UFA enriched eggs, but few have focused on the long term effects that enriched diets might have on production performances of commercial layer hens over prolonged periods of time.

The aim of this study was therefore to determine the effects of dietary fatty acid saturation on egg production performance of laying hens at end-of-lay.

\section{Materials and Methods}

Five isoenergetic (12.6 MJ AME/kg DM) and isonitrogenous (170 g CP/kg DM) diets were formulated using various supplementary lipid sources at a $30 \mathrm{~g} / \mathrm{kg}$ inclusion level to manipulate dietary fatty acid saturation levels. The control diet was formulated using a blend (50:50) of linseed- and fish oil, while pure fish oil was used in the polyunsaturated n-3 diet (PUFA n-3). In the polyunsaturated n-6 diet (PUFA n6), sunflower oil was used, whereas in the monounsaturated n-9 diet (MUFA n-9) high oleic acid (HO) sunflower oil was used as supplementary lipid source. Lastly, tallow was used as lipid source in the saturated fatty acid (SFA) treatment. Fatty acid methyl esters (FAME) of the experimental diets (Table 1) were determined by extracting the fat content thereof using the method firstly described by Folch et al. (1957). Fatty acid methyl esters were prepared for gas chromatography by methylation of the extracted fat, using methanol-BF 3 (Diaz et al., 2005) and were quantified using a Varian GX 3400 flame ionization GC, with a fused silica capillary column, Chrompack CPSIL 88 (100 m length, $0.25 \mathrm{~mm}$ ID, $0.2 \mu \mathrm{m}$ film thickness). Identified individual fatty acids were expressed as a percentage of the total fatty acids present in the sample (\% FAME).

Two hundred, individually caged Hy-Line Silver-Brown laying hens (20 weeks of age) were randomly allocated to the five dietary treatments $(n=40$ replicates/treatment) and received the experimental diets for a period of 54 weeks from 20 to 74 weeks of age. Feed and water were provided on an ad libitum basis to the birds that were individually housed in metabolism battery cages $\left(1600 \mathrm{~cm}^{2} / \mathrm{bird}\right)$.

Egg production was recorded daily for each hen and expressed as the mean hen-day egg production (\%) per week, while feed intake was determined weekly and used for the calculation of average daily feed intake (g/bird/day). During weeks 58, 62, 66, 70 and 74 of age, all eggs produced were recorded and individually weighed (g), while the body weights (g) of birds were also determined. Egg weights were used to calculate individual egg output (g/bird) of birds, whereas feed efficiency (gram egg produced/gram feed consumed) was calculated by dividing the mean egg output (g) by the mean feed intake (g). To determine the effects of dietary fatty acid saturation on performance parameters at end-of-lay, data of the collection weeks were pooled and analysed by using the one-way ANOVA procedure of the SAS ${ }^{\circledR}$ program (SAS, 2010) at a probability of $P \leq 0.05$. Tukey Kramer's studentized range (HSD) test was used to illustrate differences between treatment means as identified by the analysis of variance procedure.

\section{Results and Discussions}

The effects of dietary lipid source used on the mean fatty acid methyl esters (FAME) of the diets are indicated in Table 1. It is clearly noticeable that the FAME of the experimental diets was altered in accordance with the FAME of the specific supplementary lipid sources. The control treatment $(50: 50$ blend of linseed- and fish oil) had an increased $\alpha$-linolenic (C18:3) concentration (7.85\%), compared to that of the 
PUFA n-3 treatment (1.81\%). In contrast, the PUFA n-3 treatment (fish oil) had the highest concentration of eicosapentaenoic- (C20:5; 2.67\%), docosapentaenoic- (C22:5; 0.13\%) and docosahexaenoic acid (C22:6; $1.02 \%)$. While the PUFA n-6 treatment (sunflower oil) had the highest concentration $(41.29 \%)$ of linoleic acid (C18:2), the MUFA n-9 treatment (HO-sunflower oil) had the highest concentration (56.67\%) of oleic acid (C18:1). Lastly, the SFA treatment (tallow) had the highest concentration (16.70\%) of stearic acid (C18:0), even though the concentrations of the other main saturated fatty acids such as myristic- (C14:0) and palmitic acid (C16:0) were intermediate when compared to those FAME concentrations of the control and PUFA n-3 treatments.

Table 1 Mean fatty acid methyl esters (FAME") of the experimental diets used during weeks 58 to 74 of age.

\begin{tabular}{lrrrrr}
\hline & Control & PUFA (n-3) & PUFA (n-6) & MUFA (n-9) & SFA \\
\hline FAME $^{\#}$ (\% of total fatty acids) & & & & & \\
\hline C14:0 & 3.68 & 7.00 & 0.01 & - & 2.54 \\
C16:0 & 20.51 & 25.14 & 13.13 & 8.88 & 22.69 \\
C17:0 & 0.62 & 0.52 & 0.57 & 0.11 & 0.75 \\
C18:0 & 4.20 & 3.75 & 5.18 & 4.07 & 16.70 \\
C18:1c9 & 28.09 & 23.69 & 34.67 & 56.67 & 29.35 \\
C18:2c9,12 (n-6) & 27.05 & 23.89 & 41.29 & 26.57 & 22.98 \\
C18:3c9,12,15 (n-3) & 7.85 & 1.81 & 1.34 & 1.08 & 1.11 \\
C20:2c11,14 (n-6) & 0.49 & 0.41 & 0.84 & 0.41 & 0.18 \\
C20:5c5,8,11,14,17 (n-3) (EPA $\left.{ }^{1}\right)$ & 1.27 & 2.67 & - & - & - \\
C22:5c7,10,13,16,19 (n-3) $\left(\right.$ DPA $\left.^{2}\right)$ & - & 0.13 & - & - & - \\
C22:6c4,7,10,13,16,19 (n-3) $\left(\right.$ DHA $\left.^{3}\right)$ & 0.46 & 1.02 & - & - & - \\
\hline
\end{tabular}

${ }^{1}$ Eicosapentaenoic acid; ${ }^{2}$ Docosapentaenoic acid; ${ }^{3}$ Docosahexaenoic acid.

The effects of dietary fatty acid saturation on egg production performances at end-of-lay are summarized in Table 2. Dietary fatty acid saturation had an effect $(P=0.02)$ on the average daily feed intake of hens, whereas the SFA and PUFA n-6 diets resulted in the highest feed intake compared to the lowest feed intake of the PUFA n-3 treatment (Table 2).

Table 2 The effects (mean \pm s.d.) of dietary fatty acid saturation on egg production parameters of laying hens at end-of-lay (58-74 weeks of age)

\begin{tabular}{lcccccc}
\hline & Control & PUFA (n-3) & PUFA (n-6) & MUFA (n-9) & SFA & $P$ \\
\hline ADFI $^{1}$ (g/bird/day) & $100.7^{\mathrm{ab}} \pm 6.6$ & $97.5^{\mathrm{b}} \pm 6.2$ & $102.4^{\mathrm{a}} \pm 6.4$ & $99.9^{\mathrm{ab}} \pm 6.6$ & $102.1^{\mathrm{a}} \pm 8.7$ & 0.02 \\
Egg production $^{*}(\%)$ & 77.3 & 78.7 & 81.6 & 79.4 & 83.1 & 0.24 \\
Egg weight (g) & $61.8 \pm 4.4$ & $60.6 \pm 5.0$ & $60.6 \pm 3.5$ & $60.9 \pm 4.2$ & $59.7 \pm 4.2$ & 0.31 \\
Egg output (g) & $331.1 \pm 45.5$ & $330.2 \pm 37.7$ & $343.9 \pm 41.2$ & $336.7 \pm 40.0$ & $344.9 \pm 47.3$ & 0.39 \\
Feed efficiency (g/g) & $0.47 \pm 0.05$ & $0.48 \pm 0.05$ & $0.48 \pm 0.05$ & $0.48 \pm 0.05$ & $0.48 \pm 0.05$ & 0.71 \\
Body weight (g) & $1874 \pm 140$ & $1888 \pm 162$ & $1923 \pm 157$ & $1897 \pm 124$ & $1921 \pm 159$ & 0.54
\end{tabular}

\footnotetext{
${ }^{a, b}$ Row means with no common superscripts differ significantly $(P \leq 0.05)$;

${ }^{\#}$ Mean value for specific parameter;

${ }^{1}$ Average daily feed intake (g/bird/day).
} 
These results are difficult to explain since the diets were formulated to be isoenergetic and isonitrogenous and it is only the PUFA n-6 (sunflower oil) and SFA (tallow) treatments that resulted in the highest feed intake levels, while that of the other treatments remains similar. Partly in support to the present study, Celebi \& Macit (2008) attributed differences in feed intake of hens fed tallow and linseed oil to the effect of dietary fatty acid saturation levels on the digestibility coefficients and absorption of lipids and triglycerols, as well as the apparent metabolisable energy (AME) content of the diets. Contradictory to the present results, Cachaldora et al. (2006) reported that dietary PUFA n-3 concentration had no effect ( $P$ $>0.05$ ) on feed intake. Two possible explanations for the inconsistent results on feed intake could be (i) the variation in metabolisable energy concentration of the dietary treatments within and between studies (Gonzàlez-Esquierra \& Leeson, 2001) as well as (ii) oxidative rancidity of the lipid sources used during the different studies as suggested by Cachaldora et al. (2008).

Performance parameters presented in Table 2 revealed no significant differences between treatment means for hen-day egg production, egg weight, egg output, feed efficiency and body weights at end-of-lay (58 - 74 weeks of age). These results are supported by that of Cachaldora et al. (2006) and Cachaldora et al. (2008) who found no significant effects of dietary fatty acid saturation on egg production performance of layer hens. Conversely, Shang et al. (2004) found that body weight gain, rate of egg production, egg weight and feed efficiency decreased linearly $(P<0.01)$ with an increase in dietary fatty acid unsaturation levels during the eight week experimental period between 40 and 48 weeks of age. Yin et al. (2008) also reported a decrease in egg- and body weights with an increase in dietary UFAs during week 50 to 58 of age. The decreases in performance parameters of older hens as recorded by Shang et al. (2004) and Yin et al. (2008) could most probably been ascribed to the fact that these authors used conjugated linoleic acid (CLA) at higher inclusion levels (up to 7.8\%) to enhance the unsaturation fatty acid profile of their experimental diets. Since it is known that CLA causes weight loss in humans (Yin et al., 2008), it seems that this specific type (CLA) of unsaturated fatty acid (high in cis-9; trans-11 isomers) has a similar negative effect on body weights of laying hens and consequently egg production and egg size.

\section{Conclusion}

In general, despite the significant lowest feed intake recorded by the birds in the PUFA n-3 treatment, it seems that dietary fatty acid saturation had no significant detrimental effect on most of the production performances of laying hens during end-of-lay (58 - 74 weeks of age). Results of the present study suggest that the long-term exposure (54 weeks) to diets differing in fatty acid saturation levels had no negative effect on production performance of laying hens at end-of-lay; given the diets were formulated to be isoenergetic and isonitrogenous.

\section{Acknowledgements}

The authors wish to acknowledge Nutri Feeds, Energy oil and Vergezocht HO oils for their technical and financial support during the trial.

\section{References}

Cachaldora, P., Garcia-Rebollar, P., Alvarez, C., De Blas, J.C. \& Mendez, J., 2006. Effect of type and level of fish oil supplementation on yolk fat composition and n-3 fatty acid retention efficiency in laying hens. Br. Poult. Sci. 47, 43-49.

Cachaldora, P., Garcia-Rebollar, P., Alvarez, C., De Blas, J.C. \& Mendez, J., 2008. Effect of type and level of basal fat and level of fish oil supplementation on yolk fat composition and n-3 fatty acids deposition efficiency in laying hens. Anim. Feed Sci. Technol. 141, 104-114.

Celebi, S. \& Macit, M., 2008. The effect of sources of supplemental fat on performance, egg quality, and fatty acid composition of egg yolks in laying hens. J. Sci. Food Agric. 88, 2382-2387.

Ceylan, N., Ciftçi, I., Mizrak, C., Kahraman, Z. \& Efil, H., 2011. Influence of different dietary oil sources on performance and fatty acid profile of egg yolk in laying hens. J. Anim. Feed Sci. 20, 71-83.

Diaz, M.T., Alvarez, I., De La Fuente, J., Sanudo, C., Campo, M.M., Oliver, M.A., Font i Furnols, M., Montossi, F., San Julian, R., Nute, G.R. \& Caneque, V., 2005. Fatty acid composition of meat from typical lamb production systems of Spain, U.K., Germany and Uruguay. Meat Sci. 71, 256-263. 
Folch, J., Lees, M. \& Sloane-Stanley, G.H., 1957. A simple method for the isolation and purification of total lipids from animal tissue. J. Biol. Chem. 226, 497-509.

Gonzàlez-Esquerra, R. \& Leeson, S., 2001. Alternatives for enrichment of eggs and chicken meat with omega-3 fatty acids. Can. J. Anim. Sci. 81, 295-305.

González-Muñoz, M.J., Bastida, S., Jiménez, O., Lonrenzo, C., Vergara, G. \& Sánchez-Muniz, F.J., 2009. The effects of dietary fat on the fatty acid composition and cholesterol content of the eggs from HyLine and Warren hens. J. Poult. Sci. 60, 350-359.

Hu, F.B., Stampfer, M.J., Rimm, E.B., Manson, J.E., Ascherio, A., Colditz, G.A., Rosner, B.A., Spiegelman, D., Speizer, F.E., Sacks, F.M., Hennekens, C.H. \& Willet, W.C., 1999. A prospective study of egg consumption and risk of cardiovascular disease in men and women. J. Am. Med. Ass. 281, 1387-1394.

Leskanich, C.O. \& Noble, R.C., 1997. Manipulation of the n-3 polyunsaturated fatty acid composition of avian egg and meat. Wrld's Poult. Sci. J. 53, 155-183.

McNamara, D.J., 2000. Eggs, dietary cholesterol and heart disease risk: An international perspective. In: Egg Nutrition and Biotechnology. Eds: Sim, J.S., Nakai, S. \& Guenter, W., CABI Publishing, U.K. pp. 55-64.

SAS, 2010. Statistical analysis system $\left(\right.$ SAS $\left.^{\circledR}\right)$ user’s guide. Version 6.12. SAS Institute Inc. Cary, NC, USA.

Scheideler, S.E. \& Froning, G.W., 1996. The combined influence of dietary flaxseed level, form and storage conditions on egg production and composition among vitamin E-supplemented hens. Poult. Sci. 75, 1221-1226.

Shang, X.G., Wang, F.L., Li, D.F., Yin, J.D. \& Li, J.Y., 2004. Effects of dietary conjugated linoleic acid on the productivity of laying hens and egg quality during refrigerated storage. Poult. Sci. 83, 1688-1695.

Simopoulos, A.P., 2000. Symposium: Role of poultry products in enriching the human diet with n-3 PUFA. Poult. Sci. 79, 961-970.

Souza, J.G., Costa, F.G.P., Queiroga, R.C.R.E., Silva, J.H.V., Schuler, A.R.P. \& Goulart, C.C., 2008. Fatty acid profile of eggs of semi-heavy layers fed feeds containing linseed oil. Braz. J. Poult. Sci. 10, 37-44.

Surai, P.F., MacPherson, A., Speake, B.K. \& Sparks, N.H.C., 2000. Designer egg evaluation in a controlled trial. Eur. J. Clin. Nutr. 54, 298-305.

Yin, J.D., Shang, X.G., Li, D.F., Wang, F.L., Guan, Y.F. \& Wang, Z.Y., 2008. Effects of dietary conjugated linoleic acid on the fatty acid profile and cholesterol content of egg yolks from different breeds of layers. Poult. Sci. 87, 284-290. 